\title{
A Wearable Vibration Glove for Improving Hand Sensation in Persons with Spinal Cord Injury Using Passive Haptic Rehabilitation
}

\author{
LTC Tanya Estes, PhD \\ Assistant Professor \\ Program Director, Information Technology \\ Department of Electrical Engineering and \\ Computer Science \\ United States Military Academy \\ West Point, New York 10996 \\ Phone: (845) 938-6605 \\ Fax (845) 938-5956 \\ Email: tanya.t.estes@gmail.com
}

\author{
Deborah Backus, PT, PhD, FACRM \\ Director of Multiple Sclerosis Research \\ The Eula C. and Andrew C. Carlos Rehabilitation \\ and Wellness Program at Shepherd Center \\ 2020 Peachtree Road, NW \\ Atlanta, Georgia 30309 \\ Phone: (404) 350-7599 \\ Email: deborah_backus@shepherd.org
}

\author{
Thad Starner, PhD \\ Professor \\ Georgia Institute of Technology \\ 85 5th Street, NW \\ Atlanta, Georgia 30332 \\ Phone: (404) 385-0816 \\ Email: thad@gatech.edu
}

\begin{abstract}
We define Passive Haptic Rehabilitation (PHR) as an improvement in haptic sensations or abilities using methods which require little or no attention on the part of the user. We present a study that suggests improvement in hand sensation in participants with partial Spinal Cord Injury (SCI) in C4-T1 after use of our Mobile Music Touch (MMT) glove. The MMT glove is designed to teach piano melodies passively. It stimulates the participant's fingers repeatedly in the order of notes in the songs to be learned. In a study of ten hands, seven people with incomplete SCI participate in simple piano lessons three times a week for thirty minutes a session for eight weeks. The experimental group also attends these lessons but also wear our Mobile Music Touch (MMT) glove for two hours a day, five times a week to reinforce these lessons passively. Participants were injured over a year before the beginning of the study. The Semmes-Weinstein test is used to measure sensation at eight points on the hand before and after the piano lessons. The mean improvements between the experimental group and control group show a difference that is statistically significant. All hand areas in the experimental group show an improvement in average Semmes-Weinstein scores.
\end{abstract}

Keywords-haptic; rehabilitation; spinal cord injury; sci, vibration

\section{INTRODUCTION}

The loss or degradation of function in the hands can cause considerable loss of independence as the hands are essential to communication through writing and typing and, even more basically, the ability to feed and care for oneself. Another consideration is basic protection. If sensation is greatly degraded, one may not be aware of heat or pain, allowing for injury without the individual's awareness, potentially leading to further disability or illness. Dimitrejevic writes that "diminished afferent input to the brain from the affected hand is a common deficit after stroke. Patients become less aware of their affected upper extremity because of sensory loss and partial paralysis. As a consequence, they use that extremity less and less, learning to use the unaffected arm in its place. Over time, disuse weakens muscles and most likely reduces the representation area of the affected part in the cortex" [1]. Dimitrijevic also postulated the use of the stimulation to improve sensation in persons with spinal cord injury, which demonstrates similar loss of upper extremity use to that of persons who have suffered stroke. Similar mechanisms for many different aspects of rehabilitation are discussed by Doidge [2]. Rehabilitation after an injury or illness may allow an individual to regain use of a disabled limb.

However, rehabilitation often requires the patient to perform multiple repetitions of simple motions. While absolutely essential to regaining function, such exercises are not always mentally engaging and enjoyable for the patient. An engaging and fun form of hand rehabilitation may help encourage the patient to perform rehabilitation exercises more often. Playing instruments such as the keyboard can improve finger strength and dexterity as well as feeling of mental well-being [3]. If such rehabilitation also allows the 
patient to learn a new skill, such as playing the piano, the patient may even continue to engage in the rehabilitative exercise for life.

Most rehabilitation activities are active in nature, requiring the focused participation of the injured person. Passive rehabilitation is the idea that some technologies and activities may bring about beneficial changes without the active engagement of the injured person. Several studies have shown some possible rehabilitative benefits from haptics to bring about improvements in persons with injuries involving the central nervous system. Stimulation of the afferent (sensory) nerves can increase flexibility and range of movement and decrease muscle spasticity [1].

One possibility is that the ability to complete motor activities correctly requires feedback from the afferents in the peripheral nervous system [4]. The afferents are the nerves that take signals from the sensory organs, of which the skin is the largest in the body, and return those signals back to the brain for processing [5]. Haptic stimulation of those afferent nerves using vibration motors, for example, may help improve fine motor control of the hands due to improvements in the ability to sense or feel with the hands. We have dubbed the concept of providing rehabilitation while the recipient is engaging in an unrelated activity Passive Haptic Rehabilitation (PHR). In order to study the concept PHR, we developed the Mobile Music Touch (MMT) system (Fig. 1). This study evaluates the potential benefits of the MMT in people with chronic tetraplegia due to partial spinal cord injury (SCI).

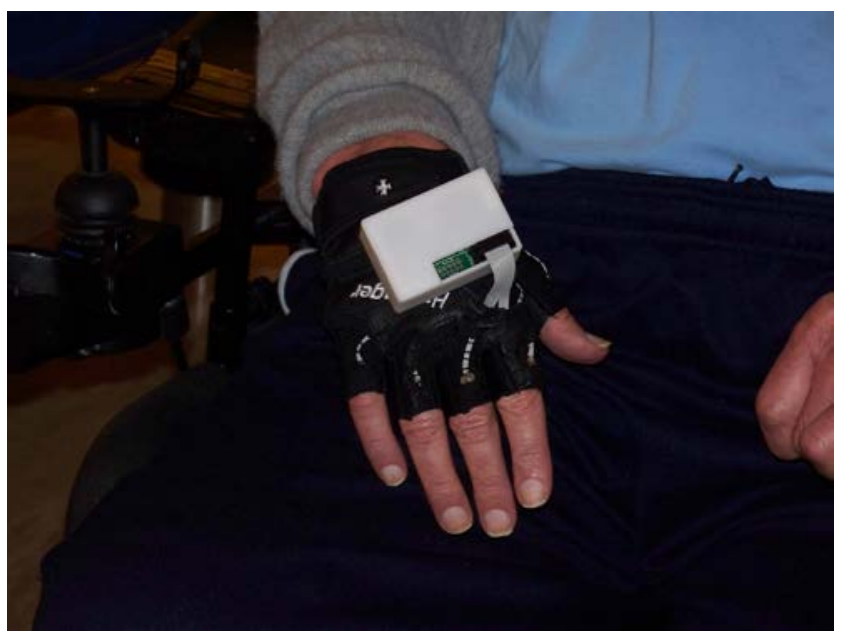

Fig. 1. The Mobile Music Touch glove uses vibration motors to stimulate each finger.

\section{MOBILE Music TOUCH}

The MMT glove is a lightweight, wireless haptic music instruction system comprised of a glove and a mobile Bluetooth-enabled computing device, such as a mobile phone. It teaches the "muscle memory" of how to play piano melodies without the learner's active attention, a process we call Passive Haptic Learning (PHL). MMT uses a fingerless glove equipped with vibrators at each knuckle. As a mobile MP3 player plays each note of a song, MMT taps the finger that corresponds to the respective piano key. In a series of controlled, randomized studies, the MMT glove successfully taught novices how to play simple, one-handed piano melodies while they were engaged by various other tasks, including completing a reading comprehension test or math exam, watching a movie, playing a memory game, completing a scavenger hunt, or reading email [6]. Surprisingly, the vibration stimulus alone is sufficient to teach these piano melodies; the audio of the music was not necessary [6]. Most recently, we have used a similar set of PHL gloves to teach a wearer how to type and read Braille in four hours without active attention.

\section{SPINAL CORD INJURY}

Spinal Cord Injury (SCI) due to injury or disease is classified by the level of injury along the spinal column [7]. An SCI can be classified as complete or incomplete. Complete SCI indicates a lesion that results in no volitional motor function or sensation below the level of injury. Incomplete SCI indicates either some sensation or voluntary movement below the point of injury, or both. In the US, there are about 12,000 new SCI each year, and about 270,000 people living in the US have SCI. The predominant group is young men, with a peak age of 19 years. The cervical cord is the most common site of injury (54\% of cases), followed by the thoracic (about 36\%), and lumbar cord (10\%) [8]. We specifically wish to work with persons who have SCI with incomplete tetraplegia. Persons with incomplete tetraplegia often have some hand function, but may have reduced sensation and motor function of the fingers and hands, and therefore the potential for meaningful improvements in function with the appropriate intervention.

In terms of preference for rehabilitation and possible outcomes, Anderson found that among persons with SCI resulting in tetraplegia, " $48.7 \%$ of the participants indicated that regaining arm and hand function would most improve their quality of life" [9], the highest ranking of all responses. Anderson's finding supports the need to focus on upper extremity rehabilitation to ensure maximum independence for persons with SCI. Upper extremity abilities dictate the level of independence of a person with SCI and must be maintained. Daily activities that require the upper extremities and are particularly crucial for people with tetraplegia are the ability to transfer from surface to surface (for example, wheelchair to standard chair or bed) [10], and to perform activities for hygiene, and eating. Beyond basic care activities, the upper extremities are key to allowing persons with SCI to communicate via phone and internet and for work and recreation. Current standard clinical practices for persons with tetraplegia include splinting [11], massed practice [12], tendon transfer surgery [13], and compensation methods using spared muscles. While these therapies are proven and effective, they are not always mentally engaging, and activities like massed practice are often discontinued after leaving the hospital.

\section{ELECTRO-STIMULATION REHABILITATION}

Dimitrijevic, Soroker, and Pollo demonstrated the use of electrical stimulation with persons suffering the effects of stroke and SCI. They took a unique approach; instead of applying electro-stim to specific points on the limb, they instead explored applying it to the entire hand. To accomplish 
this task, they had the participant wear a mesh wire glove capable of conducting electricity. To ensure even distribution of the stimulation, they also had users immerse the gloved hand in a conductive jelly. Their work produced several positive results, including a reduction in muscular spasticity, improved sensory perception in the hands, and increased range of motion in the affected upper extremity [14]. These benefits were also observed when the stimulation was below the ability of a human to detect it. Interestingly, the improvements in range of motion, spasticity, and sensation were not observed to decline even when the intervention was stopped for a month [15]. Dimitrijevic continued to explore the use of electrical stimulation applied to the afferents of the hand with Golaszewski, Kremser, Wagner, Felber, and Aichner. This team looked at the brain cortical activity of six uninjured subjects using Functional Magnetic Resonance Imaging (fMRI) performing a 20 minute finger-to-thumb tapping activity. They found that applying whole hand mesh-glove electrical stimulation (below the threshold of perception) resulted in an increase in cortical activity not seen when the sham case (no stimulation) was applied. They measured this effect by analyzing the number of activated pixels on fMRI scans (representing blood flow in the brain) when the activity was performed [16]. This study further reinforced findings from earlier stroke studies using the same mesh-glove stimulation. These studies are interesting because it appears that the electrical stimulation, even below the sensory threshold, seems to have an effect on the somatosensory system and the motor cortex.

Vibration and electrostimulation share many similarities in their perception by the human body. Might our MMT glove provide a way for the SCI user to improve sensation while learning a new skill set? In a series of preliminary experiments described in Pervasive Healthcare 2010, we performed a pilot study with two participants with tetraplegia due to partial SCI, with encouraging results [17]. Next we continued tuning our sensation and dexterity tests in able-bodied individuals. Finally, we worked with three individuals with tetraplegia to improve the design of the MMT glove for the purposes of passive rehabilitation. The results of these formative studies informed the hardware and experimental design described below.

\section{EXPERIMENT}

\section{A. Participants}

Seven individuals with SCI, levels C-4 through T-1 (6 males, 1 female) were recruited to participate in the project. Three participants qualified to complete the study with both hands, resulting in ten "hands" for the study. All participants were greater than one year post SCI, were 18-75 years of age, and able to provide consent. They also were able to perceive vibration applied to their hand, had enough ability to move their individual fingers to press the keys on a musical keyboard, and demonstrated a manual muscle test grade greater than 2, but not to total more than 10 [6] for the whole hand, in each of the following: wrist extensors, flexor profundis, and abductor minimi of the hand being tested. Participants were randomly assigned to either the "with glove" condition, wearing the MMT glove and playing piano at the research center three times a week for eight weeks, or the "without glove” condition, in which participants played piano, but did not use the MMT glove. All participants who first performed the without glove condition were given the opportunity to then participate in the with glove condition, though the extra data for these participants is not used in testing our hypothesis.

The demographics of our study participants by "hand" is depicted in Table 1; there are seven participants' data in the table, representing ten "hands," as three participants qualified for the study with both the left and the right hand. Participants complied well with the requirements of the study.

TABLE I. STUDY PARTICIPANT DEMOGRAPHICS

\begin{tabular}{|c|c|c|c|c|c|c|}
\hline Hand & Injury Date & $\begin{array}{c}\text { Level of } \\
\text { injury } \\
\text { (left/right) }\end{array}$ & Age & Sex & $\begin{array}{c}\text { Hand } \\
\text { trained }\end{array}$ & Glove \\
\hline 1 & 31 MAR 2008 & C6 & 58 & M & Right & Without \\
\hline 2 & 18 JUL 2010 & C5 & 70 & F & Right & Without \\
\hline 3 & 18 MAY 2010 & C3/C7 & 34 & M & Right & Without \\
\hline 4 & 25 MAY 2004 & C5/C6 & 26 & M & Left & Without \\
\hline 5 & 10 AUG 2009 & C5 & 24 & M & Right & Without \\
\hline 6 & 16 APR 2009 & C3/C6 & 41 & M & Right & With \\
\hline 7 & 24 NOV 2009 & C6/C7 & 29 & M & Right & With \\
\hline 8 & 25 MAY 2004 & C5/C6 & 26 & M & Right & With \\
\hline 9 & 18 JUL 2010 & C5 & 70 & F & Left & With \\
\hline 10 & 31 MAR 2008 & C6 & 58 & M & Left & With \\
\hline
\end{tabular}

\section{B. Apparatus}

The version of Mobile Music Touch (MMT) used for this study (Figs. 2 and 3) consisted of a Harbinger workout glove (Harbinger, Fairfield, CA) with vibration motors (Precision Microdrives, London, UK) fitted in each finger, and wiring and hardware mounted to the back of the hand contained in a custom 3D printed box. The MMT glove is capable of operating in two modes: controlled by a laptop or other computing device, or automatically by programming a particular song into the glove. In either mode, the MMT system stimulates, via vibration "taps," the finger that should be used to press a particular key on a piano keyboard to play notes in a song. The songs selected for this study were "pentatonic," meaning there was a one-to-one mapping for a given finger to a particular key. Using pentatonic songs minimizes and often eliminates lateral hand motion, to prevent the impact of impaired transport if proximal weakness was also present.

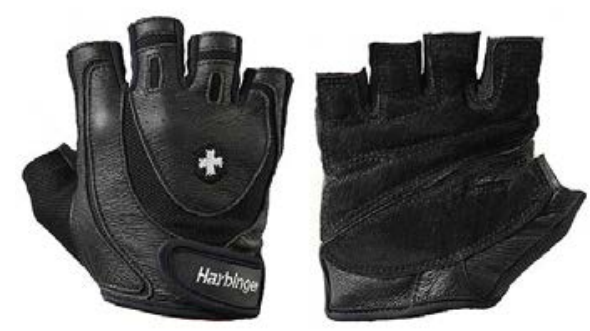

Fig. 2. Harbinger "Flex Fit” Workout Glove used in Mobile Music Touch 


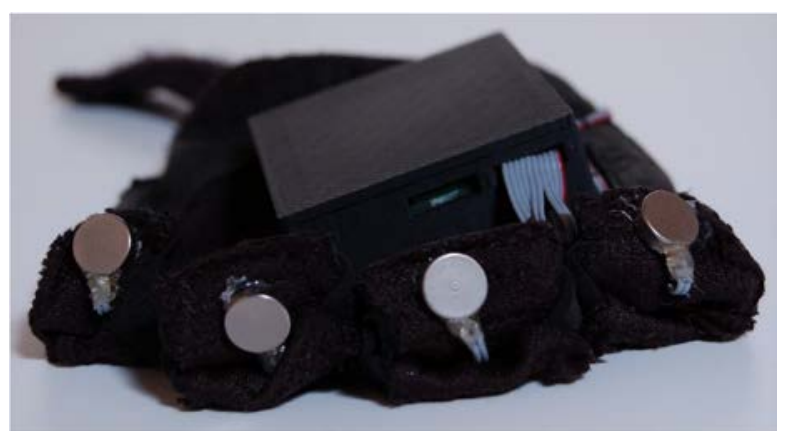

Fig. 3. MMT Glove with Vibration Motors by Precision Microdrives exposed.

\section{Units}

All participants underwent evaluations within a week of starting training (pre), at the four week midpoint (mid), and within one week after completing the training (post). Hand somatosensation was assessed using the Semmes Weinstein Monofilament Test in eight locations (see Fig. 4) and in five locations for the Two-Point Discrimination Test. Hand function was measured using the Grasp and Release Test (GRT) $[18,19,20]$ and the Action Research Arm Test (ARAT) [21]. A test of coordination and strength was provided using the Assisted Movement with Enhanced Sensation (AMES) device [22, 23]. Participants were also surveyed with poststudy questions as to their use of the glove, their enjoyment of learning piano, and their perception of the sensation and use of their hands.

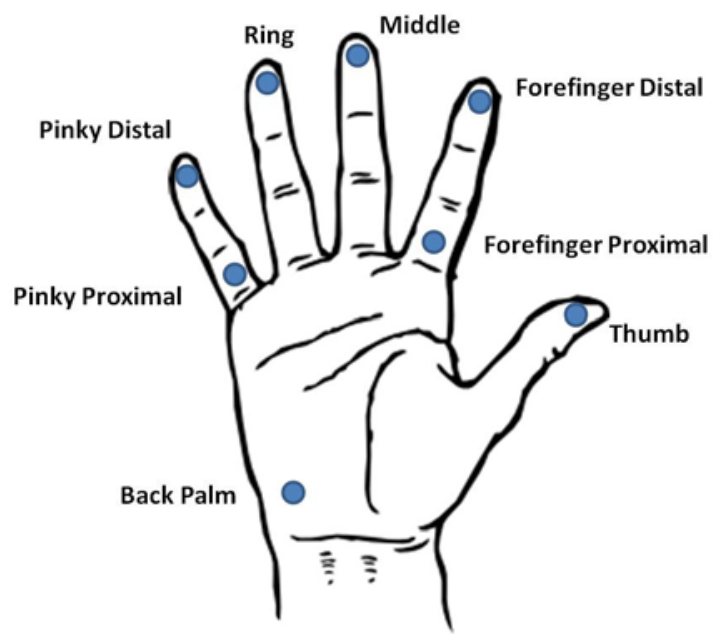

Fig. 4. Semmes-Weinstein hand test locations.

TABLE II. SEMMES-WEINSTEIN DATA

\begin{tabular}{|c|c|c|c|c|c|c|c|}
\hline \multicolumn{4}{|c|}{ Without Glove } & \multicolumn{4}{c|}{ With Glove } \\
\hline Hand & Pre & Post & Diff. & Hand & Pre & Post & Diff. \\
\hline $1 \mathrm{Rt}$ & 4.38000 & 4.38125 & 0.00125 & $6 \mathrm{Rt}$ & 4.24625 & 4.13500 & -0.11125 \\
\hline $2 \mathrm{Rt}$ & 4.34625 & 4.16500 & -0.18125 & $7 \mathrm{Rt}$ & 3.89375 & 3.68000 & -0.21375 \\
\hline $3 \mathrm{Rt}$ & 2.78125 & 2.87875 & 0.0975 & $8 \mathrm{Rt}$ & 3.31250 & 3.11250 & -0.20000 \\
\hline $4 \mathrm{Lt}$ & 2.90750 & 2.81000 & -0.09750 & $9 \mathrm{Lt}$ & 4.37750 & 3.85375 & -0.52375 \\
\hline $5 \mathrm{Rt}$ & 2.47875 & 2.58625 & 0.1075 & $10 \mathrm{Lt}$ & 4.58875 & 3.98125 & -0.60750 \\
\hline Avg. & 3.37875 & 3.36425 & -0.0145 & Avg. & 4.08375 & 3.75250 & -0.33125 \\
\hline
\end{tabular}

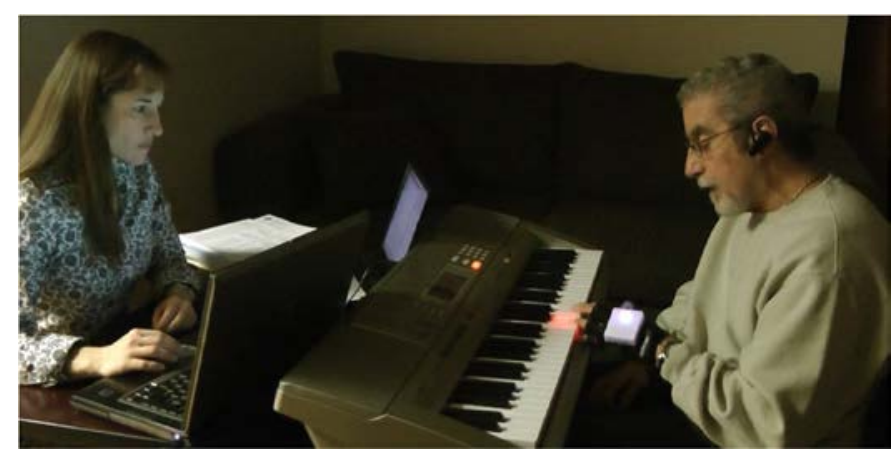

Fig. 5. Participant during an active practice session.

\section{Procedure}

The participants came to the laboratory three times a week to play the piano for 30 minutes each session, over a period of eight weeks. Each week introduced a new one-handed melody. These active practice sessions used a Casio electronic keyboard with lights in the keys. Participants watched and listened to fragments of these songs and then tried to repeat them, linking the segments into longer and longer phrases. "With glove" participants also felt the corresponding fingers stimulated when the keyboard was demonstrating how to play each segment. Fig. 5 shows a participant during one of these active practice sessions. "Without glove" participants had no additional requirements beyond just playing piano during the in-lab sessions. "With glove" participants took home an MMT glove that had the song they were working on that week programmed into the glove so that it played the song, in vibration form only, while it was turned on, with a one minute break in between each repetition of the song. They were asked to wear the glove for at least two hours a day for at least five days during the week. In order to help assure compliance with this request, the hardware also contained accelerometers and a mini SD card to record accelerometer data and time, allowing us to see that the glove was worn for the correct length of time and not sitting stationary. Participants were aware that their activity was being monitored.

\section{RESUlT}

\section{A. Somatosensation}

Using a t-test, we found that there was a significant difference between the "with glove" condition (SWmeanwith = -0.33) and the "without glove" condition (SWmeanwithout = 0.015 ) (see Fig. 6) related to the change in average SemmesWeinstein scores resulting in a p $<0.01$, 95\% confidence interval is 0.058 to 0.578 , Cohen's d: 1.78 , indicating a very high effect [24]. In this case, a reduction demonstrates an improvement in hand sensitivity, as it means the participant was able to feel a smaller diameter monofilament. SemmesWeinstein data are shown in Table 2 and Figs. 7 and 8. 


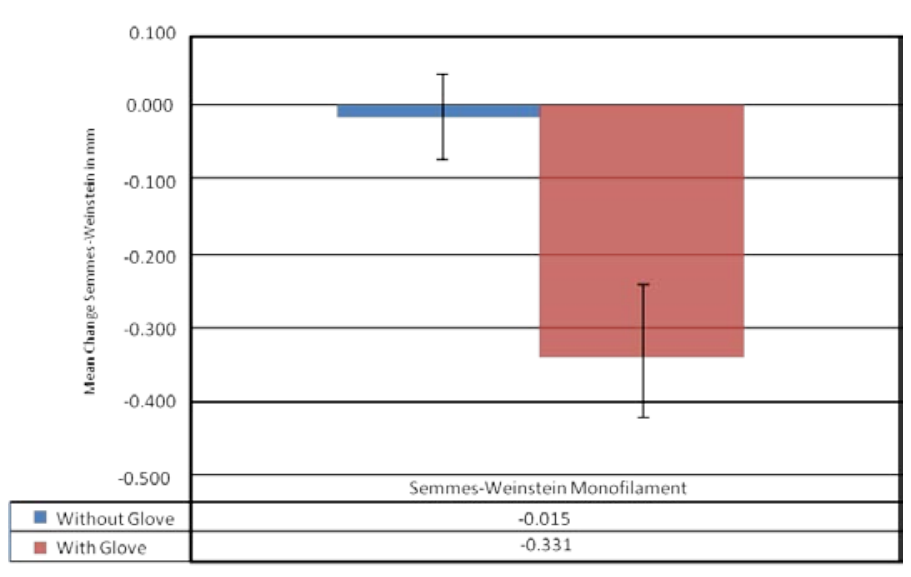

Fig. 6. Graphical depiction of Semmes-Weinstein results by condition.

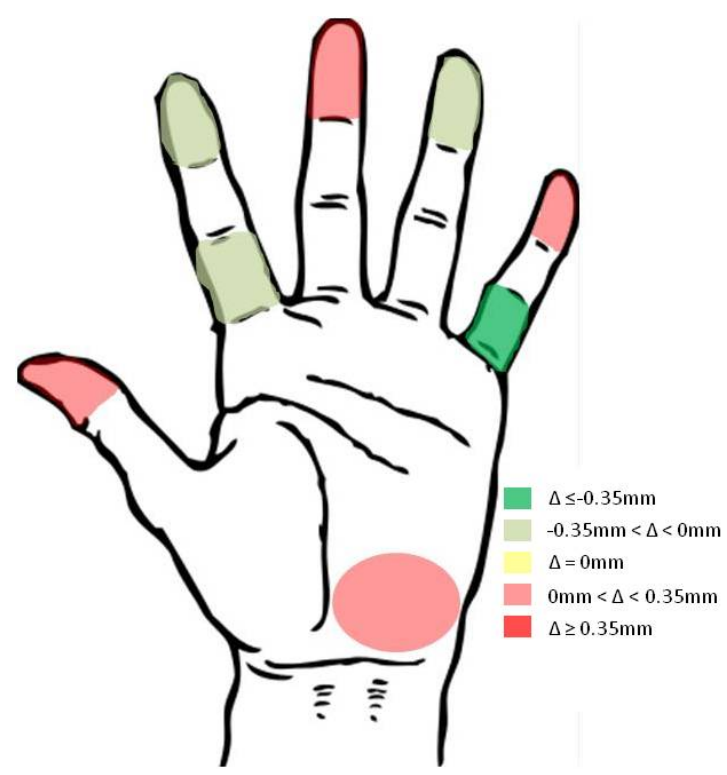

Fig. 7. Average "without glove" changes at each test site, pre to post training (smaller/greener values show improvement).

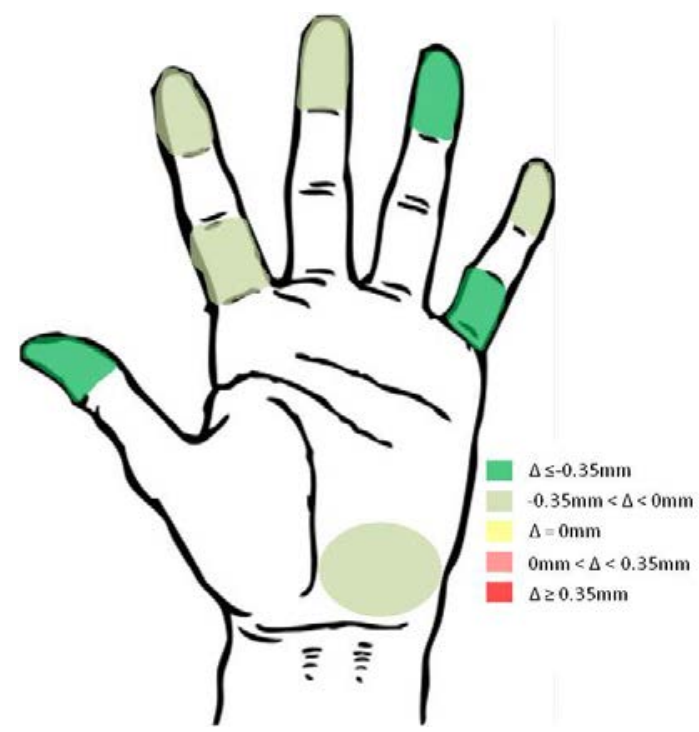

Fig. 8. Average "with glove” changes at each test site, pre to post training (smaller/greener values show improvement).

\begin{tabular}{|c|c|c|c|c|c|}
\hline & Monofilament & Hand Threshold & ID $/$ Hand & Pre & Post \\
\hline 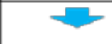 & 2.44 & \multirow{2}{*}{ Normal } & $3 \mathrm{Rt}$ & 2.78 & 2.88 \\
\hline & 2.83 & & & 2.91 & \\
\hline & 3.22 & \multirow{2}{*}{ Diminished Light Touch } & & 4.35 & 4.10 \\
\hline & 3.61 & & $1 \mathrm{Rt}$ & 4.38 & 4.831 \\
\hline & 3.84 & \multirow{4}{*}{$\begin{array}{c}\text { Diminished Protective } \\
\text { Sensation }\end{array}$} & & 2.48 & 2.59 \\
\hline & 4.08 & & & & \\
\hline & 4.17 & & & & \\
\hline & 4.31 & & & & \\
\hline & 4.56 & \multirow{2}{*}{$\begin{array}{l}\text { Loss of Protective } \\
\text { Sensation }\end{array}$} & & & \\
\hline & 4.74 & & & & \\
\hline
\end{tabular}

Fig. 9. Without glove average changes for each of the five hands, illustrated on a sensation threshold scale.

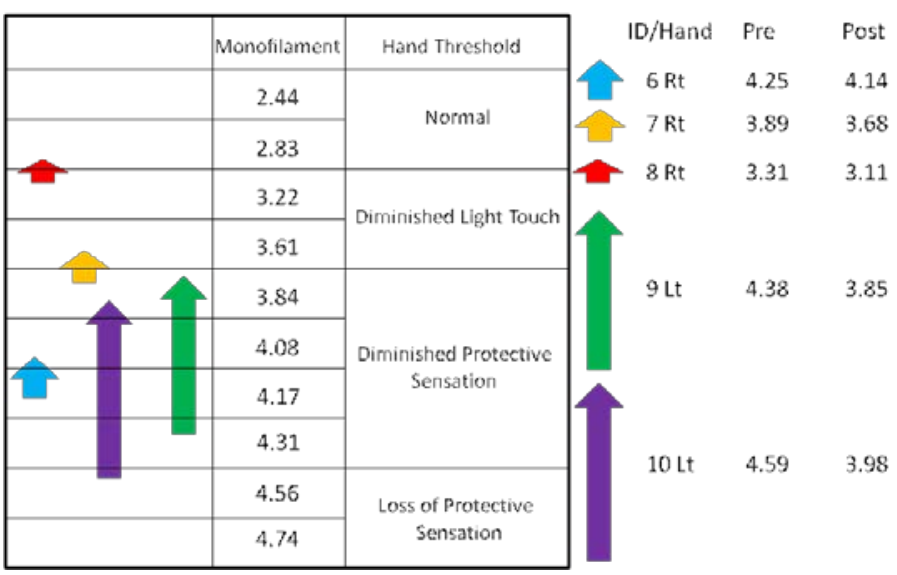

Fig. 10. "With glove" average changes for each of the five hands, illustrated on a sensation threshold scale.

Figs. 7 and 8, "without glove" and "with glove," respectively, depict the changes from pre to post training for the between subject study. Figs. 9 and 10 present the pre and 
post values to help illustrate the changes as the participants approach the goal of "Normal" on the chart. Several $(n=3$; $60 \%$ ) participants in the "with glove" group make transitions from one category of sensation to another (for example, from "Diminished Light Touch" to "Normal") while none do in the control group.

The 2-point discrimination test failed to achieve statistical significance. The means of the two groups were 2PTmeanwith $=-2.36$ for the "with glove" condition and 2PTmeanwithout $=$ 0.6 for the "without glove" condition $(p=0.15)$ (see Table 3). In this case, a reduction indicates the ability to distinguish between two points that are closer together. The 2-point discrimination test results are graphically depicted for the "without glove" condition in Fig. 11 and the "with glove" condition in Fig. 12. In the figures, green indicates an improvement in perception, yellow is no change, and red shows a reduction in perception of the two points.

TABLE III. TWO-POINT DISCRIMINATION TEST DATA

\begin{tabular}{|c|c|c|c|c|c|c|c|}
\hline \multicolumn{4}{|c|}{ Without Glove } & \multicolumn{4}{c|}{ With Glove } \\
\hline Hand & Pre & Post & Diff. & Hand & Pre & Post & Diff. \\
\hline $1 \mathrm{Rt}$ & 6.4 & 7.0 & 0.6 & $6 \mathrm{Rt}$ & 4.4 & 3.4 & -1.0 \\
\hline $2 \mathrm{Rt}$ & 12.6 & 11 & -1.6 & $7 \mathrm{Rt}$ & 11.6 & 4.2 & -7.4 \\
\hline $3 \mathrm{Rt}$ & 3.0 & 3.2 & 0.2 & $8 \mathrm{Rt}$ & 6.6 & 2.6 & -4.0 \\
\hline $4 \mathrm{Lt}$ & 5.8 & 3.6 & -2.2 & $9 \mathrm{Lt}$ & 13.2 & 14.0 & 0.8 \\
\hline $5 \mathrm{Rt}$ & 3.2 & 3.2 & 0 & $10 \mathrm{Lt}$ & 10.6 & 10.4 & -0.2 \\
\hline Avg. & 6.2 & 5.6 & -0.6 & Avg. & 9.28 & 6.92 & -2.36 \\
\hline
\end{tabular}

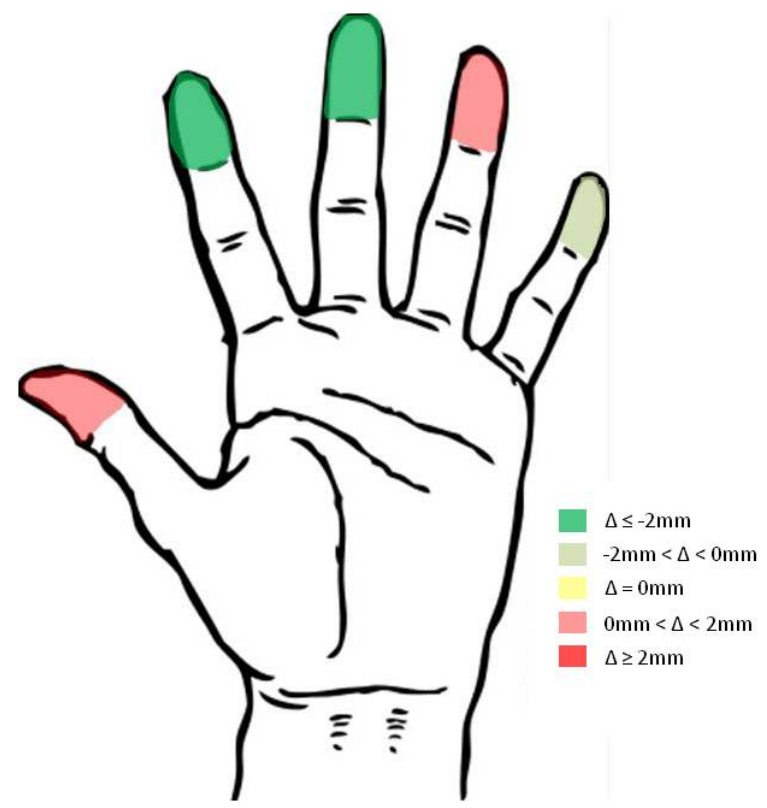

Fig. 11. Graphical depiction of 2-point discriminator test results "without glove."

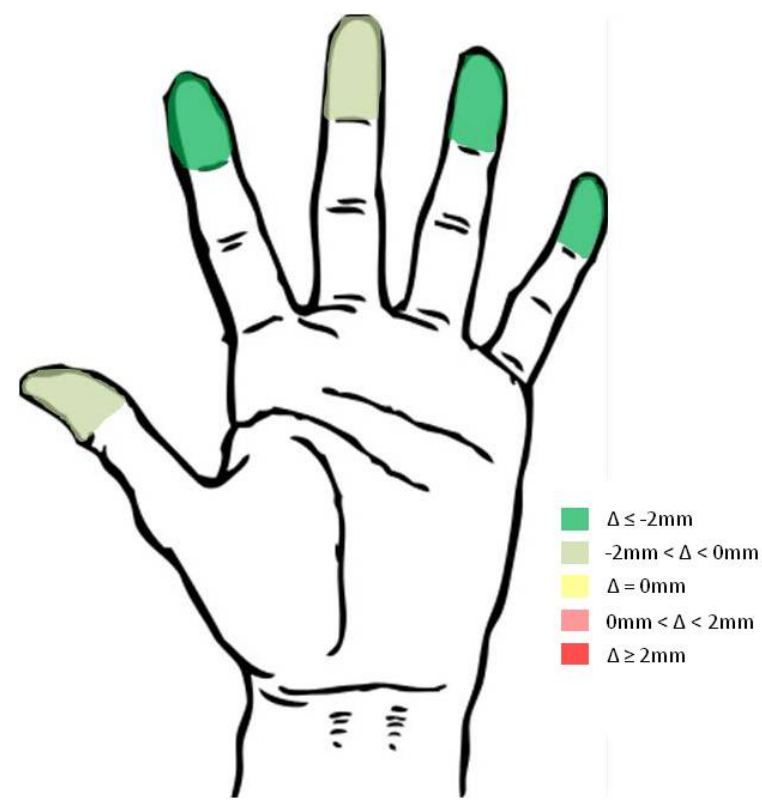

Fig. 12. Graphical depiction of 2-point discriminator test results "with glove."

\section{B. Function}

"With glove" group participants showed a significantly greater improvement on the GRT (GRTmeanwith = 29) than the "without glove" group (GRTmeanwithout $=15$ ), $\mathrm{p}=0.02$, $95 \%$ confidence interval of 0.5 through 27.5, Cohen's d: 1.55 , indicating a very high effect [24]. GRT data are shown in Table 4 and Fig. 13. In the case of the GRT, an increase represents an overall increase in the number of correctly completed repetitions of a given activity in the test. Thus, a positive change in value is considered desirable in this test.

TABLE IV. GRT TEST DATA

\begin{tabular}{|c|c|c|c|c|c|c|c|}
\hline \multicolumn{5}{|c|}{ Without Glove } & \multicolumn{4}{|c|}{ With Glove } \\
\hline Hand & Pre & Post & Diff. & Hand & Pre & Post & Diff. \\
\hline $1 \mathrm{Rt}$ & 145 & 164 & 19 & $6 \mathrm{Rt}$ & 238 & 264 & 26 \\
\hline $2 \mathrm{Rt}$ & 164 & 165 & 1 & $7 \mathrm{Rt}$ & 154 & 176 & 22 \\
\hline $3 \mathrm{Rt}$ & 158 & 175 & 17 & $8 \mathrm{Rt}$ & 164 & 204 & 40 \\
\hline $4 \mathrm{Lt}$ & 211 & 220 & 9 & $9 \mathrm{Lt}$ & 97 & 129 & 32 \\
\hline 5 Rt & 200 & 229 & 29 & $10 \mathrm{Lt}$ & 182 & 207 & 25 \\
\hline Avg. & 175.6 & 190.6 & 15 & Avg. & 167 & 196 & 29 \\
\hline
\end{tabular}




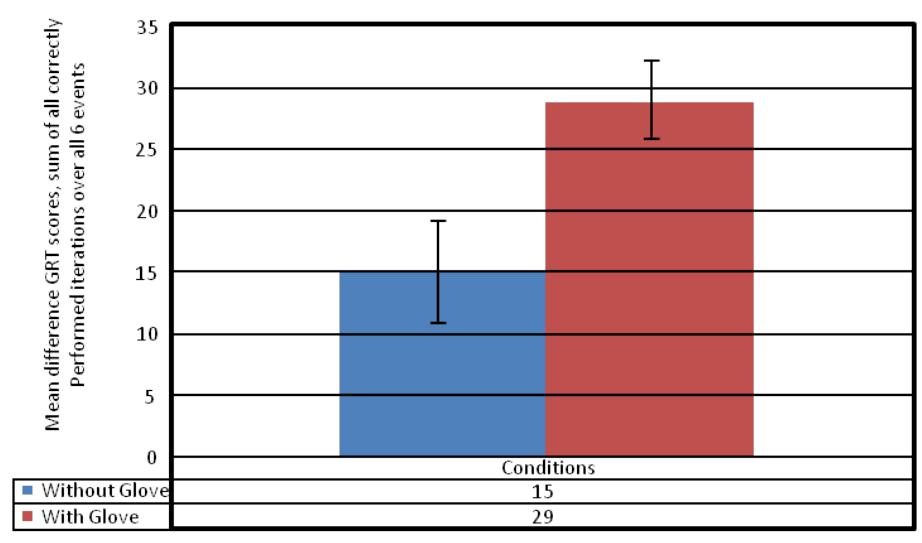

Fig. 13. Graphical depiction of Grasp and Release Test results by condition.

Although there was a positive trend in the ARAT scores, particularly in the case of the pinch sub-scale, the means did not show a statistically significant difference.

\section{DISCUSSION}

The above results suggest that use of the MMT glove for the active and passive practice of piano melodies may result in improved sensation and function for certain people with tetraplegia due to partial spinal cord injury. The increase in the number of correctly performed repetitions on the GRT suggests an improved use of the hand for opening and closing, making it easier to handle objects. In the case of our participant population, we had several who performed at or near the maximum scores possible on the ARAT. We anticipated this possibility, as in order to qualify for our study, participants had to be able to activate the keys of the piano with each finger. Our inclusion criteria require a level of hand use that causes them to come close, to, or achieve, the maximum score on the ARAT. Thus, our results on the ARAT may be suffering from a ceiling effect.

The changes in somatosensation and not strength (given the AMES test results) suggest that the sensory changes are influencing hand and wrist control, which could lead to improvements in activities of daily living, such as eating and dressing. In fact, participants in the with glove group did selfreport improvements in using items like doors, dishes, keys, and letter openers. However, the control group also reported some improvements (with keyboards, scissors, knives, and soda cans), possibly due to a rehabilitative effect from the active piano practice.

In our post-questionnaire, both groups enjoyed playing the piano and perceived hand improvement in terms of sensation and motor abilities. Both groups also reported a general improvement in mood over the course of the study. Participants who wore the glove reported a higher degree of satisfaction with piano playing improvement than those participants who did not. The without glove group also did see a slightly higher difficulty in mastering a given song in one week. The with glove group had a higher percentage feel they had learned a new skill (not just performing rehab) and felt that the glove aided in learning the songs. The glove did not interfere with participants' daily activities, was comfortable, and did not cause embarrassment. Interestingly, the with glove group also reported that the vibration did not interfere with daily activities, and that they found they tapped along with the song or could "hear" the song as it played in vibration form on their hand.

One can make the argument that the participants may have been actively moving their fingers during their "passive" wearing of the MMT glove. We chose an experimental design that valued ecological validity over internal validity in that we hoped to test the MMT glove in realistic, everyday situations. If the participants were actively manipulating their fingers while using the gloves (against advice to the contrary) and reaping a benefit from it over a two month period, perhaps the average user will as well? In any case, the experiment suggests that the MMT glove was wearable in our participants' daily lives, effective, and may make a convenient, at-home, mobile rehabilitation aid.

\section{FUTURE WORK}

With 10 participating hands, this experiment is relatively small. The next step is to repeat the experiment, increasing the number of participants. Given the intended population, we will have to increase the geographic range and participating centers significantly to scale the study to 100 hands. Here, we did not characterize the SCI in our participants. The levels of injury varied, as did the completeness of the injury. Therefore, the possibility exists that certain types or levels of injury may respond better to this approach. This possibility cannot be explored with the current data but warrants further investigation. Being able to predict a priori the amount of improvement we can expect for a given individual would help significantly in determining whether to recommend the device. We also wish to explore using the MMT glove for other types of injury (for example, stroke, traumatic brain injury, and multiple sclerosis) and exploring Passive Haptic Rehabilitation for other parts of the body.

\section{CONCLUSION}

Participants with tetraplegia due to partial spinal cord injury who used the Mobile Music Touch glove in active and passive practice of piano melodies demonstrated a greater degree of improvement in sensitivity and fine motor control of the hands than those who only performed active practice. Further study is warranted to determine the range of benefits possible and the people who are most likely to benefit from passive rehabilitation. 


\section{ACKNOWLEDGMENT}

This material is based upon work supported by the National Science Foundation under Grant No. 1217473.

\section{REFERENCES}

[1] M.M. Dimitrijevic, N. Soroker, and F.E. Pollo. "Mesh glove electrical stimulation," in Science \& Medecine, vol. 3, no. 3, 1996

[2] N. Doidge, The Brain that Changes Itself. New York, NY: Viking, 2007.

[3] C.M. Zelazny. "Theraputic instrumental music playing in hand rehabilitation for older adults with osteoarthritis: four case studies," in Journal of Music Therapy, vol. 38, no. 2, pp. 97-113, 2001.

[4] Cordo P, Lutsep H, Cordo L, Wright W, Cacciatore T, Skoss R. Assisted Movement with Enhanced Sensation (AMES): Coupling motor and sensory to remediate motor deficits in chronic stroke patients. Neurorehabilitation \& Neural Repair. vol. 23, pp. 67-77, 2008.

[5] Hoffman L, Field-Fote E. Cortical reorganization following bi-manual training and somatosensory stimulation in cervical spinal cord injury. Physical Therapy. vol. 87, pp. 208-223, 2007.

[6] Markow, T. "Mobile Music Touch: Using Haptic Stimulation for Passive Haptic Rehabilitation and Learning”, Ph.D. dissertation, College of Computing, Georgia Institute of Technology, Atlanta, GA, 2012.

[7] “Spinal Cord 101.” http://www.spinalinjury.net, February 2012.

[8] J. Little, B. Goldstein, M. Hammond. "Spinal Cord Injury," in Office of the Surgeon General, Department of the Army, United States of America, 1998.

[9] K. Anderson, "Targeting Recovery: Priorities of the spinal cord-injured population," in Journal of Neruotrauma, vol. 21, no. 10, 2004.

[10] G. Snoek, M. Ijzerman, H. Hermans, D. Maxwell, F. Biering-Sorensen, "Survey of the needs of patients with spinal cord injury: impact and priority for improvement in hand function in tetraplegics," in Spinal Cord, vol. 42, 2004.

[11] M. Curtin, "Development of a tetraplegic hand assessment and splinting protocol," in Paraplegia, vol. 32, 1994.

[12] K. Beekhuizen, E. Field-Fote, "Sensory stimulation augments the effects of massed practice training in persons with tetraplegia," in Archives of Physical Medicine and Rehabilitation, vol. 89, 2008.

[13] A. Freehafter, E. Vonhaam, V. Allen, "Tendon transfers to improve grasp after injuries of the cervical spinal cord," in Journal of Bone \& Joint Surgery, vol. 56, 1974.

[14] M. Dimitrijevic, D. Stokic, A. Wawro, C. Wun, "Modification of motor control of wrist extension by mesh-glove electrical afferent stimulation in stroke patients," in Archives of Physical Medicine and Rehabilitation, vol. 77, pp. 252-258, 1996.

[15] M. Dimitrijevic, "Mesh-glove: A Method for whole-hand electrical stimulation in upper motor neuron dysfunction," in Scandinavian Journal of Rehabilitation Medicine, vol. 26, pp. 183-186, 1994.

[16] S. Golaszewski, C. Kremser, M. Wagner, S. Felber, F. Aichner, M. Dimitrijevic, "Functional magnetic resonance imaging of the human motor cortex before and after whole-hand afferent electrical stimulation,” in Scandinavian Journal of Rehabilitation Medicine, vol. 31, pp. 165-173, 1999.

[17] T. Markow, N. Ramakrishnan, K. Huang, T. Starner, M. Eicholtz, S. Garrett, H. Profita, A. Scarlata, C. Schooler, A. Tarun, "Mobile Music Touch: Vibration Simulus in Hand Rehabilitation," in Pervasive Computing Technologies for Healthcare (PervasiveHealth), 2010.

[18] M. Mulcahey, D. Hutchinson, S. Kozin, "Assessment of upper limb in tetraplegia: Considerations in evaluation and outcomes research," in Journal of Rehabilitation Research \& Development, vol. 44, pp. 91-102, 2007.

[19] P. Taylor, J. Esnouf, J, Hobby, "The functional impact of the freehand system on tetraplegia hand function, clinical results," in Spinal Cord, pp. 560-566, 2002

[20] L. Harvey, J. Batty, R. Jones, J. Crosbie, "Hand function of c6 and c7 tetraplegics 1-16 years following injury," in Spinal Cord, vol. 39, pp. 37-43, 2001.

[21] C. Lang, J. Wagner, A. Dromerick, D. Edwards, "Measurement of upper-extremity function early after stroke: properties of the action research arm test," in Archives of Physical Medicine and Rehabilitation, vol. 87, no. 12, pp. 1605-1610, 2006.

[22] D. Backus, P. Cordo, A. Gillott, C. Kandilakis, M. Mori, A. M. Raslan, "Assisted movement with proprioceptive stimulation reduces impairment and restores function in incomplete spinal cord injury," in Archives of physical medicine and rehabilitation, 2014.

[23] S. Wolf, J.S. Lou, R. Bogey, J. Hayes, E. Roth, P.J. Cordo, "AMES Treatment of Severe Hand Impairment Following Stroke," in Stroke, vol. 44, 2013.

[24] J. Cohen, Statistical Power Analysis for the Behavioral Sciences $\left(2^{\text {nd }}\right.$ ed.), Hillsdale, NJ: Lawrence Earlbaum Associates, 1988. 